\title{
Editorial: Biofunctional Applications of Zirconia in Dental Devices
}

\author{
Guang Hong ${ }^{1 *}$, James Kit-Hon Tsoi ${ }^{2}$ and Jan-Min Han ${ }^{3}$ \\ ${ }^{1}$ Division for Globalization Initiative, Graduate School of Dentistry, Tohoku University, Sendai, Japan, ${ }^{2}$ Department of Dental \\ Biomaterials, Faculty of Dentistry, The University of Hong Kong, Hong Kong, China, ${ }^{3}$ Department of Dental Materials, School \\ of Stomatology, Peking University, Beijing, China
}

Keywords: zirconia, dentistry, biocompatibility, mechanical properties, surface properties

\section{Editorial on the Research Topic}

\section{Biofunctional Applications of Zirconia in Dental Devices}

In dentistry, esthetics is one of the main goals regardless of whether other properties of the material are insufficient. Zirconia, especially Y-TZP, has gained popularity due to its mechanical, esthetic, and outstanding aesthetic performance.

The Research Topic on "Biofunctional Applications of Zirconia in Dental Devices" in Frontiers in Dental Medicine includes two Reviews and two mini reviews that summarize recent advances on the dental application of zirconia and give research prospect guidance to solve existing problems.

Zirconia is an ideal material for dental and orthopedic application, with its excellent mechanical properties, chemical stability, biocompatibility, and negligible thermal conductivity. In recent years, it has been reported that zirconia implants are a promising alternative to titanium implants, exhibiting similar osseointegration but a superior soft-tissue response in short-term outcomes. The current reviews provide a pertinent summary of the advances in this field of research. Lin et al. review the effects of $\mathrm{ZrO}_{2}$ on soft tissues. Ectopic epithelial cells have been observed on the surface of $\mathrm{ZrO}_{2}$, suggesting that $\mathrm{ZrO}_{2}$ may be a promising material that can enhance the adhesion of epithelial cells. This review also provides an insight on the application of zirconia as an implant. It can be concluded that: 1 . Zirconia is biologically safe when used as an implant material, 2. It has an osteogenic effect when used in bone grafts, 3. It has an affinity to the surrounding soft tissues, 4. New molding technologies have been developed, and 5. Zirconia surfaces can be modified to improve biological activity. Furthermore, the review also weighs the advantages and disadvantages of the use of zirconia in the dental field.

Although zirconia-based bioceramics for dental implants have been used for at least 30 years, some limitations still exist, such as manufacturing difficulties, low temperature degradation (LTD), long-term stability, and clinical experience. Researchers continue to try various ways to solve existing problems, some limited by both lab-based and clinical research. In their mini review, Sun et al. explain the three different crystal structures of zirconia and summarized the currently feasible zirconia modification methods and divided them into three parts, namely physical treatment, chemical treatment, and coatings. At the same time, an intuitive table shows the surface modification methods and lists the effects in enhancing bone formation.

The pursuit of a more esthetic monolithic zirconia without affecting durability has become a driving force for the development of materials in the dental research community. But there is still a query on which laboratory test method is best for revealing and quantifying the useful life of clinical titanium and zirconia. Qu and Liu focused on preclinical and clinical evolutions between titanium and zirconium. In their mini review, they summarize the methods used to test both materials in 
both laboratory and clinical studies and highlight the inability to provide adequate proof on the comparability of the two materials due to lack of long-term follow ups.

Artificial Intelligence (AI) is attracting considerable interest and is rapidly developing because of computer technology. Based on data analyses and prospective evaluation, AI techniques enhance zirconia-related research. Luo et al. in their review display a clear vision in AI in dental and zirconia-related research, including clinical usage and laboratory-based studies. With the use of AI, color matching, customizing abutments, and improvement in cementation techniques can be addressed. This reduces the errors of manufacture and thus the cost of prosthodontic therapy. The review also introduced some interesting concepts, such as AI appointments, medical guidance, oral disease diagnosis, and oral monitoring, which related to many hot clinical topics. And a special clinical research on gum inflammation diagnosing which used Deeplabv3+ network with Xception and mobilNetV2 was listed, greatly confirming the feasibility of AI in the future. Through this review, we can see the evolution of AI in the development of novel approaches for the biomedical applications of zirconia in dental devices and get a clear understanding of AI technology.

In conclusion, with this Research Topic we have received a series of reviews describing the progress made in the biofunctional applications of zirconia. These reviews greatly contribute to giving a further understanding of new dental materials, as well as an outstanding viewpoint of potential usage in dental devices.

\section{AUTHOR CONTRIBUTIONS}

$\mathrm{GH}$ drafted and further revised the manuscript. JT and J-MH critically reviewed the manuscript and significantly improved it. All authors listed have read this editorial and approved it for publication.

\section{ACKNOWLEDGMENTS}

As Research Topic editors we would like to express our sincere thanks to all authors and references for their valuable contributions, which made it possible for us to complete this interesting Research Topic on Biofunctional Applications of Zirconia in Dental Devices.

Conflict of Interest: The authors declare that the research was conducted in the absence of any commercial or financial relationships that could be construed as a potential conflict of interest.

Publisher's Note: All claims expressed in this article are solely those of the authors and do not necessarily represent those of their affiliated organizations, or those of the publisher, the editors and the reviewers. Any product that may be evaluated in this article, or claim that may be made by its manufacturer, is not guaranteed or endorsed by the publisher.

Copyright (c) 2022 Hong, Tsoi and Han. This is an open-access article distributed under the terms of the Creative Commons Attribution License (CC BY). The use, distribution or reproduction in other forums is permitted, provided the original author(s) and the copyright owner(s) are credited and that the original publication in this journal is cited, in accordance with accepted academic practice. No use, distribution or reproduction is permitted which does not comply with these terms. 